\title{
Slow light between two absorbing resonance in asymmetry double quantum dots
}

\author{
YU ChunChao ${ }^{1,2} \&$ WANG Tao ${ }^{1 *}$ \\ ${ }^{1}$ Wuhan National Laboratory for Optoelectronics, Huazhong University of Science and Technology, Wuhan 430074, China; \\ ${ }^{2}$ College of Physical Science and Technology, Yangtze University, Jingzhou 434023, China
}

Received October 10, 2012; accepted December 25, 2012

\begin{abstract}
We demonstrate the slow light in double quantum dots (QDs) resonance dispersion material in theory. The slow factor, absorption, and bandwidth are greatly influenced by the energy difference of the two resonance energy. The bandwidth of this system is up to $60 \mathrm{GHz}$. The $20 \mathrm{ps}$ input signal pulse is delayed by $180 \mathrm{ps}$ (group index of approximately 55) relative to free-space propagation with little broadening in $1 \mathrm{~mm}$ dispersion material for the optical communication wavelength. The signal pulse delay can be tuned by the pump pulse.
\end{abstract}

slow light, bandwidth, slow factor, absorption coefficient

Citation: $\quad$ Yu C C, Wang T. Slow light between two absorbing resonance in asymmetry double quantum dots. Chin Sci Bull, 2013, 58: 2418-2422, doi: 10.1007/ s11434-013-5875-2

Recently, slow light attractive for the delay line has been studied widely in the quantum dots (QDs) systems. Most early work is based on electromagnetically induced transparency (EIT) [1-8]. The QDs homogeneous line width $[1,2]$, inhomogeneous broadening $[1,5,6,8]$, many-body interactions [3], size and geometry [4] dependence on the slow light properties of QDs is studied. The QDs EIT absorption dip is also observed in [7]. The delay-bandwidth product of EIT in gases as well as QDs is studied in [8]. However, the slow factor is greatly reduced by the temperature and inhomogeneous broadening. Simultaneously, slow light using coherent population oscillations (CPO) in QDs at room temperature [9-13] is researched due to their three-dimensional confinement potential. However, the delay-bandwidth product is not satisfied in the practical optical communication. And also, slow light in QD photonic crystal waveguides is researched [14]. Slow light based on tunneling induced transparency is reported in [15]. The slow and fast light in semiconductor waveguides including QDs is reviewed in [16].

Simultaneously, for a double resonance in the atomic

*Corresponding author (email: wangtao@hust.edu.cn) vapor or fiber [17-23], the possibility of the pulse delay has also received some attention. Especially, 1.6 G-bandwith, tunable, multiple-pulse-width optical delays in cesium vapor for $852 \mathrm{~nm}$ signal pulse is reported in [21]. The pulse distortion is low, the bandwidth is large, and the pulse delay is long. It is potential to be applied in the optical communication. However, there is no study on the double absorbing resonance in QDs. It may be interesting for that the QDs' resonance energy can be adjusted, and the bandwidth of the slow light may be enlarged for the optical communication.

In this work, we study the slow light in the double resonance asymmetry QDs systems. It shows a wider bandwidth, a more compact dimension, compatible for the optical communication. And the pulse delay can be tuned by the pump pulse.

\section{Model and theory}

The asymmetrical double QDs system shown in Figure 1(a) consists of two kinds of dots with different band structures shown in Figure 1(b). The absorption spectra of QDs are expected to be a series of $\delta$-function-like discrete lines due 


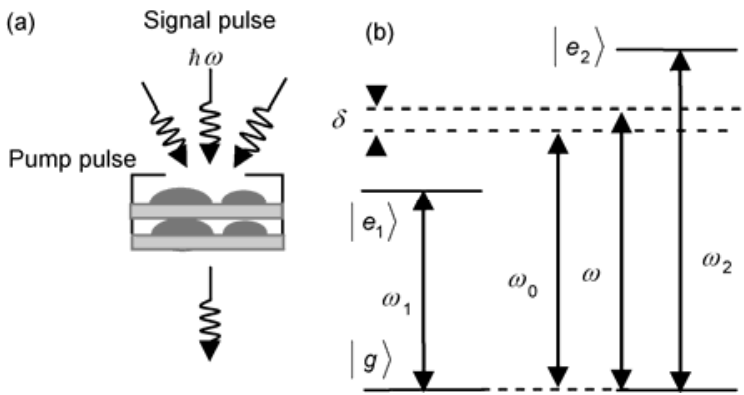

Figure 1 (a) Schematic of the system. An optical signal pulse transits the QDs systems and the pump pulse pumps the QDs to the excited states. (b) Schematic band structure and level configuration of a double QD system for the two absorbing resonance. The QD's resonance energy is little different from each other and can be controlled by the shape or a little bias voltage. A pulse laser phone energy lies between the double QDs resonance energy. $|g\rangle$ is the system state without excitations, and $\left|e_{1}\right\rangle\left(\left|e_{2}\right\rangle\right)$ is a pair of electron and hole state in the first (second) dot.

to the nature of state density. The resonance energy of the QDs is relevant to the shape and composition of QDs $[24,25]$. Therefore, these two QDs have resonance energy different from each other, indicated by $\hbar \omega_{1}$ and $\hbar \omega_{2}$.

And there are two pump pulses with the photon energy $\hbar \omega_{1}$ and $\hbar \omega_{2}$ which can pump QDs into the excited states.

At low temperature, we did not consider the inhomogeneous broadening. We defined $\omega_{0}=\left(\omega_{1}+\omega_{2}\right) / 2, \quad \omega_{21}=\left(\omega_{2}-\right.$ $\left.\omega_{1}\right) / 2, \quad \omega=\omega_{0}+\delta . \quad \delta$ is the detuning from $\omega_{0}$. All are shown in Figure 1(b).

Firstly, we consider a QD as two-level system. The laser field only coupled the vacuum and direct exciton states, with Rabi frequency $\Omega=\mu E / 2 \hbar$, where $\mu$ is the dipole momentum matrix element and $E$ is the electric field amplitude. The dielectric constant is a complex function that contains all the information about the coherent optical excitations. The macroscopic dielectric constant near the signal frequency is related to the density matrix element $\rho_{01}$ by [1]

$$
\varepsilon_{1}(\omega)=\varepsilon_{\mathrm{bac}}+\frac{2 \mu}{\varepsilon_{0} E} \frac{\Gamma}{V} \rho_{01},
$$

where $\Gamma$ is the optical confinement factor, $V$ is the volume of a single QD, and $\varepsilon_{0}$ is the dielectric constant.

By calculating the density matrix equation [26,27], we finally get

$$
\rho_{01}(\omega)=\frac{E \mu}{\hbar \omega_{1}-\hbar \omega-i \hbar \gamma},
$$

where $\gamma$ is the dephasing rate. From eqs. (1) and (2), we can get

$$
\varepsilon_{1}(\omega)=\varepsilon_{\mathrm{bac}}+\frac{2 \mu^{2}}{\hbar \varepsilon_{0}} \frac{\Gamma}{V} \frac{1}{\omega_{1}-\omega-i \gamma}=n_{\mathrm{bac}}^{2}+\frac{U}{\omega_{1}-\omega-i \gamma},
$$

where $\varepsilon_{\mathrm{bac}}=n_{\mathrm{bac}}^{2}, \quad \varepsilon_{0} \varepsilon_{\mathrm{bac}}$ is the background dielectric con- stant without coupling to any light. $U=2 \Gamma\left|\mu_{10}\right|^{2} /\left(\hbar \varepsilon_{0} V\right)$.

Finally, the complex refractive index can be written as

$$
\begin{aligned}
\tilde{n}_{1} & =\sqrt{\varepsilon_{1}}=\left(n_{\mathrm{bac}}^{2}+\frac{U}{\omega_{1}-\omega-i \gamma}\right)^{1 / 2} \\
& \approx n_{\mathrm{bac}}+\frac{U}{2 n_{\mathrm{bac}}} \frac{1}{\omega_{1}-\omega-i \gamma} .
\end{aligned}
$$

Therefore, the effective complex refractive index of our asymmetry double QDs can be written [23]:

$$
\tilde{n}(\omega)=n_{\mathrm{bac}}+\frac{U}{2 n_{\mathrm{bac}}}\left(\frac{g_{1}}{\omega_{1}-\omega-i \gamma}+\frac{g_{2}}{\omega_{2}-\omega-i \gamma}\right),
$$

where $g_{1}$ and $g_{2}$ account for the possibility of different strengths for the two resonance. In the following, we assume $g_{1}=g_{2}=1 / 2$ for the error introduced is little [21]. Using the defined above, we can get

$$
\tilde{n}(\delta)=n_{\mathrm{bac}}-\frac{U}{4 n_{\mathrm{bac}}}\left(\frac{1}{\delta+\omega_{21}+i \gamma}+\frac{1}{\delta-\omega_{21}+i \gamma}\right) .
$$

We can calculate the slow factor and the absorption coefficient from eq. (5) [28]:

$$
\begin{gathered}
S=\frac{c}{v_{\mathrm{g}}}=\operatorname{Re}(\tilde{n})+\omega \frac{\partial \operatorname{Re}(\tilde{n})}{\partial \omega}, \\
\alpha(\omega)=\frac{2 \omega}{c} \operatorname{Im}(\tilde{n}) .
\end{gathered}
$$

From eqs. (7) and (8), the slow factor and absorption coefficient of the system are calculated:

$$
\begin{gathered}
S=n_{\mathrm{bac}}+\frac{U \omega}{4 n_{\mathrm{bac}}}\left\{\frac{\left(\omega-\omega_{1}\right)^{2}-\gamma^{2}}{\left[\left(\omega-\omega_{1}\right)^{2}+\gamma^{2}\right]^{2}}+\frac{\left(\omega-\omega_{2}\right)^{2}-\gamma^{2}}{\left[\left(\omega-\omega_{2}\right)^{2}+\gamma^{2}\right]^{2}}\right\}, \\
\alpha(\omega)=\frac{\omega U \gamma}{2 n_{\mathrm{bac}} c}\left[\frac{1}{\left(\omega_{1}-\omega\right)^{2}+\gamma^{2}}+\frac{1}{\left(\omega_{2}-\omega\right)^{2}+\gamma^{2}}\right] .
\end{gathered}
$$

At $\omega=\omega_{0}$, we can get

$$
\begin{gathered}
S_{0}=S\left(\omega_{0}\right)=n_{\mathrm{bac}}+\frac{U \omega_{0}}{4 n_{\mathrm{bac}}}\left[\frac{\omega_{21}^{2}-\gamma^{2}}{\left(\omega_{21}^{2}+\gamma^{2}\right)^{2}}+\frac{\omega_{21}^{2}-\gamma^{2}}{\left(\omega_{21}^{2}+\gamma^{2}\right)^{2}}\right], \\
\alpha_{0}=\alpha\left(\omega_{0}\right)=\frac{\omega U \gamma}{n_{\mathrm{bac}} c \omega_{21}^{2}} .
\end{gathered}
$$

Pulse propagation can be described by the various orders of the dispersion, which can be calculated from

$$
\beta_{n}(\omega)=\frac{\partial^{n}}{\partial \omega^{n}}[\omega \operatorname{Re}(\tilde{n})]
$$

When the slow light is put into practical applications, it is important to achieve large delays over significant band- 
width and in a compact device. The obstacles include the loss, the group velocity dispersion and the absorption dispersion. The bandwidth of the double QDs systems is limited by two factors [23]. The first is the third-order dispersion. The criteria is

$$
\left(\Delta \omega_{1 / 2}\right)_{d}=8 \ln 2 B<\left(\frac{32 \ln 2}{\left|\beta_{3}\right| L}\right)^{1 / 3}=\left(\frac{32 \ln 2 n_{\mathrm{bac}} c \omega_{21}^{4}}{3 \omega_{0} U L}\right)^{1 / 3},
$$

where $B$ is a bit interval. The second is the absorption dispersion, and the criteria is

$$
\left(\Delta \omega_{1 / 2}\right)_{a}<\left[\frac{32(\ln 2)^{3}}{3 \alpha_{0} L}\right]^{1 / 2} \omega_{21} .
$$

The bandwidth of the double QDs resonance system is the minor of $\left(\Delta \omega_{1 / 2}\right)_{d}$ and $\left(\Delta \omega_{1 / 2}\right)_{a}$. When the pulse transits the material, there is pulse broadening because of the dispersion and absorption. And the main cause is the dispersion [21]. We can calculate the broadening of the pulse only considering the dispersion by

$$
T_{d}=\sqrt{T_{0}^{2}+\left(\frac{3 \alpha_{0} L}{2 \gamma \omega_{21}^{2} T_{0}^{2}}\right)^{2}},
$$

where $T_{0}$ is the initial pulse width.

For a Gauss pulse $\Psi_{\mathrm{G}}(0, t)=\Psi_{0} \exp \left[-\left(t / 2 T_{0}\right)^{2}\right]$, when it transits the material, the output pulse is proportional to $[19,21,23]$

$$
\Psi_{\mathrm{G}}(L, t) \propto \Psi_{0} \exp \left[-\alpha_{0} L-\frac{1}{4}\left(t-t_{d}\right)^{2} / T_{d}^{2}\right],
$$

where $\Psi_{0}$ is the amplitude of pulse at the input.

\section{Results and discussion}

According to InGaAsP/InP QDs, we can choose the parameters as follows: $n_{\mathrm{bac}}=3.2, \varepsilon_{0}=8.85 \times 10^{-12} \mathrm{~F} / \mathrm{m}, \Gamma=6 \times 10^{-3}$, $V=1.82 \times 10^{-24} \mathrm{~m}^{3}, \hbar r=2 \mu e v, \mu=6 \times 10^{-29} \mathrm{C} \mathrm{m}, \omega_{0}=2 \pi \times 1.935$ $\times 10^{14} \mathrm{rad} / \mathrm{s}[29,30]$.

Firstly, we can calculate the refractive index and the group velocity by eqs. (6) and (7). It is shown in Figure 2. When the photon energy approximates the resonance energy of each QD, there is large refractive dispersion due to the single QD refractive dispersion. Though there is large slow factor near the resonance energy, the signal may be distorted because of the large refractive dispersion and the bandwidth is small. When the signal energy is just lied in the middle of the two QDs resonance energy, there is a wide range of the frequency where the group velocity dispersion is relatively small. We can get a large bandwidth with small dispersion. The group velocity is $0.018 c$ when the detuning

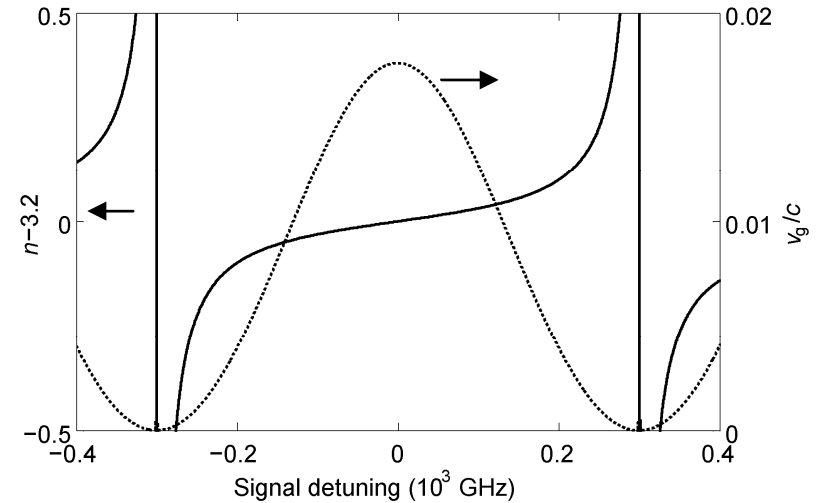

Figure 2 The refractive index of the double QDs system and the reciprocal of the slow factor versus probe detuning. $\omega_{21}=2 \pi \times 3 \times 10^{11} \mathrm{rad} / \mathrm{s}$.

is zero.

Therefore, we especially pay attention to the little dispersion in the middle of the double QDs resonance energy. Little dispersion means a large bandwidth with little distortion accompanied by maximum transparency. However, at the same time, the slow factor also is the smallest. The energy between the two resonance energy has a great influence on the slow light factor, absorption coefficient and the bandwidth. Figure 3 shows the absorption index of the double QDs system and the reciprocal of the slow light factor versus a half of the two resonance energy difference at zero detuning described by eqs. (11) and (12). The absorption coefficient declines and the reciprocals of the slow light factor go up with the increasing of the energy difference. Lower absorption means lower loss, but at the same time, the slow light factor also is small, meaning a little delay for the signal pulse. Considering the tradeoff of the absorption and the slow factor, we choose $\omega_{21}=2 \pi \times 3 \times 10^{11} \mathrm{rad} / \mathrm{s}$, just as Figure 1.

The bandwidth of the double QDs resonance system is determined by eqs. (14) and (15). In Figure 3, the bandwidth declined as the rising of the material length. In our double QDs resonance system, the bandwidth is mainly

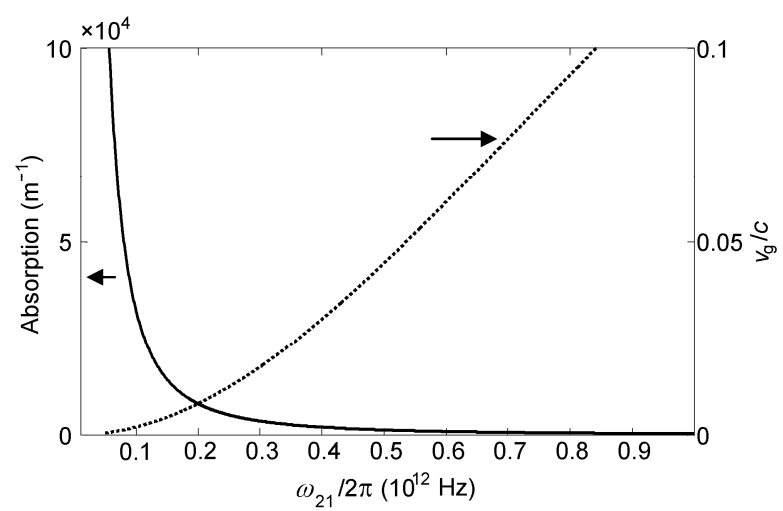

Figure 3 The absorption index of the double QDs system and the reciprocal of the slow light factor versus a half of the two resonance energy difference at zero detuning. 


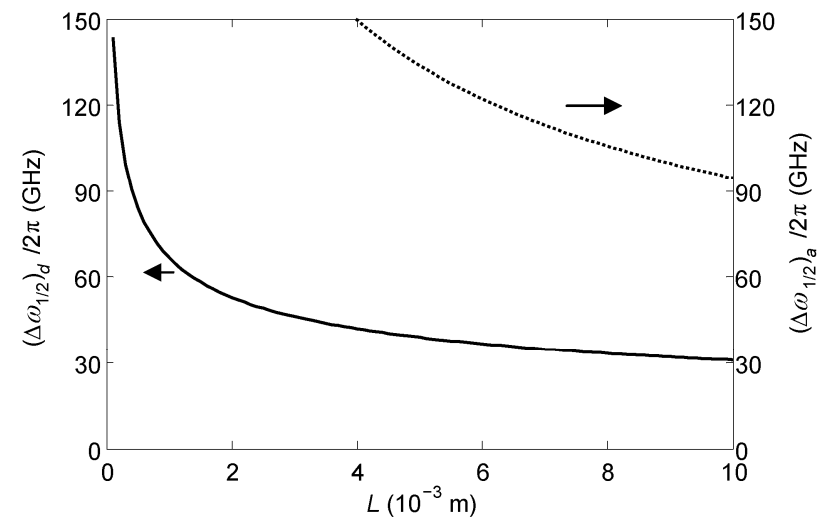

Figure 4 The bandwidth limited by the dispersion and absorption versus the material length.

decided by the third-order dispersion. The bandwidth is about $60 \mathrm{GHz}$ for $1 \mathrm{~mm}$ material, which is much larger than the slow light based on EIT [1]. The bandwidth approaches $30 \mathrm{GHz}$ with the increase of the material length.

From eq. (16), the signal broadening can be calculated. As to an input signal $T_{0}=20 \mathrm{ps}$, we finally get $T_{d} \approx 20.01 \mathrm{ps}$ and the time delay of the signal is about $180 \mathrm{ps}$ for $1 \mathrm{~mm}$ dispersion material. Therefore the signal broadening can be neglected in this work. In Figure 5, the signal transiting the dispersion material is simulated by eq. (17). For $1 \mathrm{~mm}$ dispersion material, signal broadening is very little. The deficiency is the loss which is about $-15 \mathrm{~dB}$ for $1 \mathrm{~mm}$ dispersion material.

Finally, we also can tune the delay rapidly by optically pumping the QDs into the excited state. As shown in Figure 1 , each pump laser is resonant with one of the QDs transition in order to saturate the QDs without optical pumping from one hyperfine level to the other. And the pump pulse absorption is much larger than the signal pulse. The maximum transmission length is smaller than the dispersion material thickness, so there is only a portion of the dispersion material joining in the two absorbing resonance. The pump

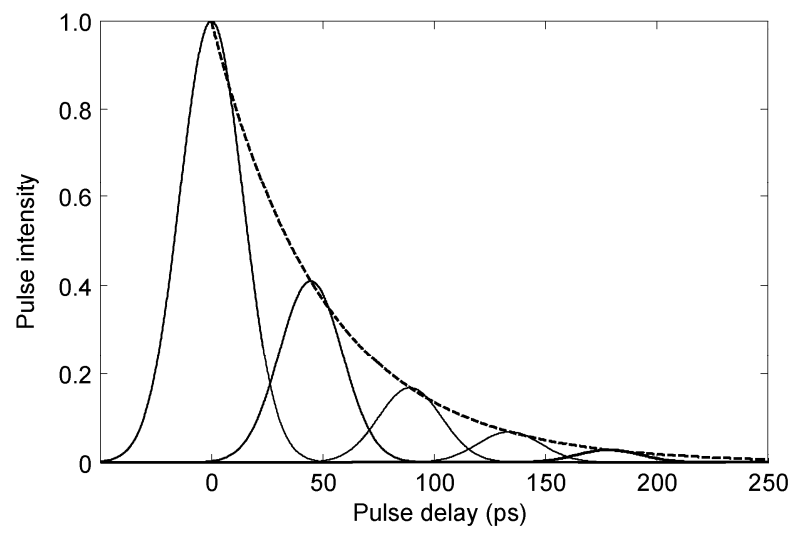

Figure 5 Pulse shapes of 20 ps Gauss input pulses transiting the double QDs resonance system versus the pulse delays with different material length. The dashed line is the transmission of the signal. pulses transit the dispersion material with the pulse power $I=I_{0} e^{-\alpha L}$, where $\alpha$ is the absorption coefficient, and $I_{0}$ is the pump light power. Obviously, the effective pump length in the dispersion material is related to the pump power and it can be tuned by the pump pulse power. $L \infty \ln I_{0}$. The signal pulse delay is

$$
t_{\text {delay }}=\frac{L}{v_{\mathrm{g}}} \propto \ln I_{0} .
$$

Therefore, we can tune the signal pulse delay quickly by changing the pump pulse power.

\section{Conclusions}

In this paper, we theoretically investigate the slow light in double QDs resonance dispersion material. In the middle of the two resonance energy, the refractive dispersion is relatively small where it shows a large bandwidth for optical communication. The energy difference of the two resonance energy determines the slow factor, the absorption, and the bandwidth. The bandwidth of this system reaches $60 \mathrm{G}$ and the signal pulse is delayed by many pulse widths relative to free-space propagation with little broadening in $1 \mathrm{~mm}$ dispersion material for optical communication wavelength. The signal pulse delay can be tuned by the pump pulse.

This work was supported by the National Basic Research Program of China (2010CB923204), National High Technology Research and Development Program of China (2008AA03Z404) and National Natural Science Foundation of China (60877040).

1 Ku P C, Chang-Hasnain C J, Kim J, et al. Variable optical buffer using slow light in semiconductor nanostructures. Proc IEEE, 2003, 91 : 1884-1897

2 Nielsen P K, Thyrrestrup H, Mørk J, et al. Numerical investigation of electromagnetically induced transparency in a quantum dot structure. Opt Express, 2007, 15: 6396-6408

3 Houmark J, Nielsen T R, Mørk J, et al. Comparison of electromagnetically induced transparency schemes in semiconductor quantum dots tructures: Impact of many-body interactions. Phys Rev B, 2009, 79: 115420

4 Barettin D, Houmark J, Lassen B, et al. Optical properties and optimization of electromagnetically induced transparency in strained InAs/GaAs quantum dot structures. Phys Rev B, 2009, 80: 235304

5 Lunnemanna P, Mørk J. Reducing the impact of inhomogeneous broadening on quantum dot based electromagnetically induced transparency. Appl Phys Lett, 2009, 94: 071108

6 Lunnemann P, Mørk J. A scheme comparison of Autler-Townes based slow light in inhomogeneously broadened quantum dot media. J Opt Soc Am B, 2010, 27: 2654-2664

7 Marcinkevičius S, Gushterov A, Reithmaier J P. Transient electromagnetically induced transparency in self-assembled quantum dots. Appl Phys Lett, 2008, 92: 041113

8 Tidström J, Jänes P, Andersson L M. Delay-bandwidth product of electromagnetically induced transparency media. Phys Rev A, 2007, 75: 053803

9 Chang S W, Chuang S L. Slow light based on population oscillation in quantum dots with inhomogeneous broadening. Phys Rev B, 2005, 72: 235330 
10 Mørk J, Kjær R, van der Poel M, et al. Slow light in a semiconductor waveguide at gigahertz frequencies. Opt Express, 2005, 13: 8136-8145

11 Matsudaira A, Lee D, Kondratko P, et al. Electrically tunable slow and fast lights in a quantum-dot semiconductor optical amplifier near $1.55 \mu \mathrm{m}$. Opt Lett, 2007, 32: 2894-2896

12 Kondratko P K, Chang S W, Su H, et al. Optical and electrical control of slow light in p-doped and intrinsic quantum-dot electroabsorbers. Appl Phys Lett, 2007, 90: 251108

13 Chen Y H, Mørk J. Enhancing slow- and fast-light effects in quantum-dot semiconductor waveguides through ultrafast dynamics. Opt Lett, 2010, 35: 697-699

14 Nielsen T R, Lavrinenko A, Mørk J. Slow light in quantum dot photonic crystal waveguides. Appl Phys Lett, 2009, 94: 113111

15 Borges H S, Sanz L, Villas-Bôas J M, et al. Tunneling induced transparency and slow light in quantum dot molecules. Phys Rev B, 2012, 85: 115425

16 Mørk J, Lunnemann P, Xue W, et al. Slow and fast light in semiconductor waveguides. Semicond Sci Tech, 2010, 25: 083002

17 Camacho R M, Pack M V, Howell J C. Low-distortion slow light using two absorption resonances. Phys Rev A, 2006, 73: 063812

18 Camacho R M, Broadbent C J, Ali-Khan I, et al. All-optical delay of images using slow light. Phys Rev Lett, 2007, 98: 043902

19 Camacho R M, Pack M V, Howell J C. Low-distortion slow light using two absorption resonances. Phys Rev A, 2006, 73: 063812

20 Zhu Z M, Gauthier D J. Nearly transparent SBS slow light in an optical fiber. Opt Express, 2006, 14: 7238-7245
21 Camacho R M, Pack M V, Howell J C, et al. Wide-bandwidth, tunable, multiple-pulse-width optical delays using slow light in cesium vapor. Phys Rev Lett, 2007, 98: 153601

22 Shakhmuratov R N, Odeurs J. Slow light with a doublet structure: Underlying physical processes and basic limitations. Phys Rev A, 2008, 77: 033854

23 Khurgin J B. Slow light in various media: A tutorial. Adv Opt Photon, 2010, 2: 287-318

24 Cusack M A, Briddon P R, Jaros M. Absorption spectra and optical transitions in InAs/GaAs self-assembled quantum dots. Phys Rev B, 1997, 56: 4047-4050

25 Zhang B B, Liu X H, Li D, et al. Preparation of multi-color quantum dots and its application to immunohistochemical analysis. Chin Sci Bull, 2008, 53: 2077-2083

26 Cakir B, Yakar Y, Özmen A. Linear and nonlinear refractive index changes in spherical quantum dot. Prog Electromagn Res M, 2011, 21: 71-92

27 Wang G H, Guo K X. Interband optical absorptions in a parabolic quantum dot. Physica E, 2005, 28: 14-21

$28 \mathrm{Wu}$ J, Li Y P, Yang C C, et al. Slow light in tapered slot photonic crystal waveguide. Chin Sci Bull, 2009, 54: 3658-3662

29 Borri P, Langbein W, Schneider S, et al. Ultralong dephasing time in InGaAs quantum dots. Phys Rev Lett, 2001, 87: 157401

30 Al-Khakani M K, Al-Mossawi K H, Al-Khursan A H. Slow light using InGaAsP semiconductor quantum dot systems. Phys Express, 2011, 1: 199-207

Open Access This article is distributed under the terms of the Creative Commons Attribution License which permits any use, distribution, and reproduction in any medium, provided the original author(s) and source are credited. 